\title{
APPLICATION DE LA THÉORIE DU CHAMP ÉLECTROMAGNÉTIQUE AUX MACHINES LINÉAIRES A INDUCTION
}

\author{
par Robert BONNEFILLE et Michel KANT \\ Laboratoire de Génie Electrique de la Faculté des Sciences de Paris $\left({ }^{*}\right)$ \\ et Laboratoire Central des Industries Electriques, 33 avenue du Général-Leclerc, \\ 92, Fontenay-aux-Roses
}

(Reçu le 15 mai 1970)

\begin{abstract}
Résumé. - On développe une théorie générale de la machine linéaire à induction, commune aux dispositifs à induits solide et liquide, en mentionnant cependant les problèmes d'analyse spécifiques à chacun de ces systèmes. On étudie plus particulièrement les effets de pénétration du champ magnétique dans un induit homogène en mouvement, les phénomènes liés aux configurations particulières des circuits magnétiques et électriques, les possibilités d'élimination des effets parasites et d'optimalisation énergétique des machines linéaires.
\end{abstract}

\begin{abstract}
A general theory of the linear induction machine with solid or liquid armature is developped including discussion of the analytical problems specific to each of these two types.

More particulary, study is made of the effects of the magnetic field penetration in an homogenous moving armature, of the phenomena related to the various configurations of magnetic and electrical circuits, of the possibilites of eliminating parasitic effects, and of the possible energy optimisation of the linear machine.
\end{abstract}

1. Introduction. - Une machine linéaire à induction, utilisée comme convertisseur d'énergie électrique en énergie mécanique ou inversement, se compose généralement de deux inducteurs plans, comportant des enroulements d'excitation polyphasés et d'un induit constitué soit par une bande métallique solide (moteur linéaire) soit par un conduit véhiculant le métal liquide (pompe ou générateur).

Le principe de fonctionnement est celui du couplage magnétique asynchrone : les enroulements d'excitation créent un champ magnétique glissant qui engendre dans l'induit (équivalent à la partie rotorique d'une machine tournante classique) des f. é. m., donc des courants. L'interaction des courants induits et du champ inducteur se traduit par une poussée qui est motrice lorsque la vitesse de déplacement $V$ de l'induit est inférieure à celle $V_{\mathrm{c}}$ du champ, et résistante dans le cas contraire.

L'analogie de principe entre une machine tournante et une machine linéaire à induction ne doit pas faire

(*) Laboratoire associé au C. N. R. S. oublier les caractères spécifiques de cette dernière, et notamment :

- l'inducteur, ouvert aux deux extrémités,

- l'entrefer, large et dont la majeure partie est occupée par l'induit, conducteur,

- l'induit lui-même constitué, par une masse conductrice, généralement homogène et isotrope, et très différent de l'induit tournant où des conducteurs isolés transportent les courants dans des directions imposées.

La géométrie très particulière de la machine linéaire, imposant une puissance spécifique inférieure à celle de la machine tournante, se traduit également par des conditions de fonctionnnement différentes ; par exemple, le glissement des machines linéaires est généralement supérieur à celui des dispositifs tournants, et leur rendement est donc théoriquement plus faible.

Les machines linéaires sont essentiellement caractérisées par une faible réactance de magnétisation, une faible inertie mécanique, une excellente capacité thermique et une évidente simplicité de réalisation; c'est dans les domaines où de telles propriétés se traduisent par des avantages certains que ces machines 
doivent trouver d'intéressantes applications, d'autant plus qu'elles peuvent se substituer à des dispositifs électromécaniques complexes.

Bien que le principe des machines linéaires soit connu depuis très longtemps, les études théoriques les concernant sont très récentes et essentiellement liées aux noms de Shturman [1], [2], Laithwaite [3] et Voldeck [4], [5], [6].

2. Description élémentaire de la machine. 2.1 Constitution GÉNÉRAle (*). - Quel que soit le dispositif envisagé, nous l'étudierons en considérant le schéma de la figure 1 qui représente une coupe transversale de la machine, rapportée à un triède trirectangle $O x y z$ : le déplacement du champ glissant et de la partie active de l'induit s'effectuant suivant l'axe longitudinal $O x$, la composante principale de l'induction magnétique $B$ est dirigée suivant l'axe transversal $O y$. Selon cet axe $O y$ et de part et d'autre de $O x$ on peut distinguer plusieurs couches caractérisées électriquement par leur perméabilité $\mu$ et leur résistivité $\rho$ :



FIG. 1.

- la couche 1 , d'épaisseur $2 a$, seule mobile, forme la partie active de l'induit; elle est conductrice $\left(10^{-8} \Omega . \mathrm{m}<\rho_{1}<10^{-5} \Omega . \mathrm{m}\right)$ et généralement non magnétique $\left(\mu_{1} \approx \mu_{0}\right)$;

- la couche 2 , non magnétique $\left(\mu_{2} \approx \mu_{0}\right)$, de faible épaisseur $b$, est constituée soit par une zone d'air (moteur linéaire $; \rho_{2} \approx \infty$ ), soit par des parois plus ou moins isolantes (dispositifs M. H. D.) ;

- la couche $2^{\prime}$, également non magnétique $\left(\mu_{2} \approx \mu_{0}\right)$ et de très faible épaisseur $b^{\prime}$, n'existe que dans les dispositifs M. H. D. où elle joue le rôle d'isolant électrique et thermique $\left(\rho_{2}, \approx \infty\right)$;

- la couche 3 , d'épaisseur $h$, formant la carcasse magnétique, est constituée de tôles feuilletées $\left(\rho_{3} \approx \infty\right)$ de haute perméabilité $\mu_{3}$.

(*) Nous considérons que l'induit de la machine est mobile et les inducteurs fixes, ce qui est le cas des dispositifs M. H. D. et de certains moteurs linéaires, mais les conclusions de cette étude sont également valables pour les machines à inducteurs mobiles et à induit fixe.
2.2 InduCteurs. - Dans les enroulements d'excitation des inducteurs plans, circulent des courants de direction $O z$ déterminés par la configuration des encoches; le champ d'induction $\mathbf{B}$ correspondant a pour composante $\left(B_{x}, B_{y}, 0\right)$.

Afin de diminuer la complexité des calculs des phénomènes magnétiques, nous étudions le champ dans l'entrefer à l'échelle du pas polaire en faisant abstraction des détails à l'échelle du pas dentaire (alternance des encoches et des dents). Pour représenter les courants statoriques dans une machine ainsi " géométriquement lisse ", les enroulements d'excitation sont remplacés par des nappes infiniment minces en chaque point desquelles lest /définie une densité linéique de courant $J_{\mathrm{c}}$; en désignant par

$$
\omega=2 \pi f
$$

la pulsation des courants inducteurs et par $\mathbf{u}_{z}$ le vecteur unitaire de l'axe $O z, \mathbf{J}_{\mathrm{c}}$ est de la forme

$\mathbf{J}_{\mathbf{c}}=\left[\mathbf{u}_{z} f(x) \mathrm{e}^{j \omega t}\right]$ pour $y= \pm\left(a+b^{\prime}+b\right)$.

L'amplitude de $\mathbf{J}_{c}$ est liée à celle des courants de phases, $I_{c}$, par la relation classique des machines électriques

$$
J_{\mathrm{c}}=\frac{I_{\mathrm{c}} m_{f} \cdot n}{p \lambda}
$$

avec $m_{f}$ - nombre de phases, $n-$ nombre de conducteurs efficaces en série par phase, $p$ - nombre de paires de pôles, $\lambda$-longueur d'onde ou double pas polaire.

Signalons que cette représentation équivalente correspond à une approximation assez grossière qui ne permet de connaître les phénomènes magnétiques ni dans les inducteurs ni dans leur voisinage immédiat. Remarquons également que la relation (1) suppose un branchement direct des deux inducteurs, c'est-àdire que le rôle du deuxième est simplement de doubler la f. m. m. de la machine ; il n'en est pas toujours ainsi et, pour certaines pompes électromagnétiques par exemple [7], il convient de considérer deux nappes inductrices dont les densités de courant présentent un décalage temporel.

2.3 InDUIT. - Si la conception électrique de l'inducteur d'un moteur linéaire et d'un dispositif M. H. D. est sensiblement identique, celle de l'induit est fort différente.

L'induit d'un moteur linéaire peut être assimilé à une bande conductrice solide, de largeur $c$ et d'épaisseur $2 a$ (Fig. 2a) située dans le champ magnétique principal, et complétée par deux bandes de largeur $d$, également conductrices, disposées généralement en dehors de ce champ. L'ensemble des trois bandes se déplace à la vitesse constante $\mathrm{V}\left(V_{x}, 0,0\right)$ par rapport aux inducteurs. 


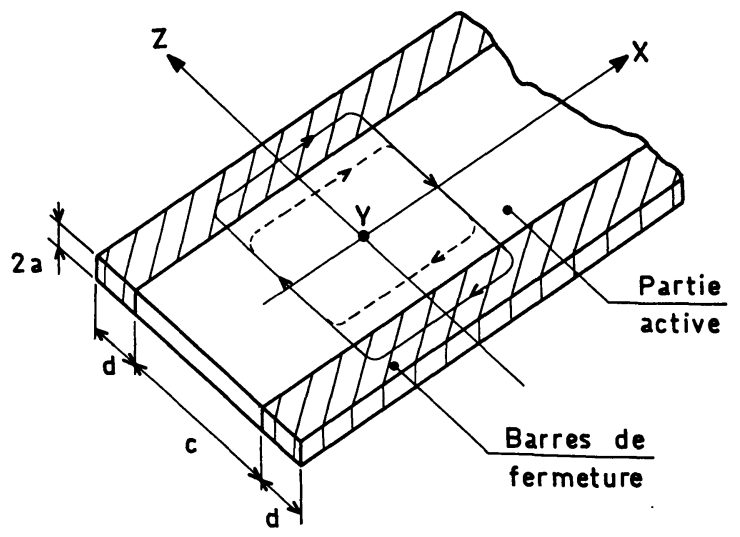

FIG. $2 a$.

L'induit d'un dispositif M. H. D. se compose de trois parties : un conduit fixe contenant le métal liquide mobile, ce dernier constituant la partie active conductrice, de largeur $c$ et d'épaisseur $2 a$ (Fig. $2 b$ ); deux barres conductrices de largeur $d$ situées généralement à l'extérieur du champ magnétique principal et formant les parois latérales du conduit; deux parois horizontales du conduit, d'épaisseur $b$, entièrement à l'intérieur du champ.

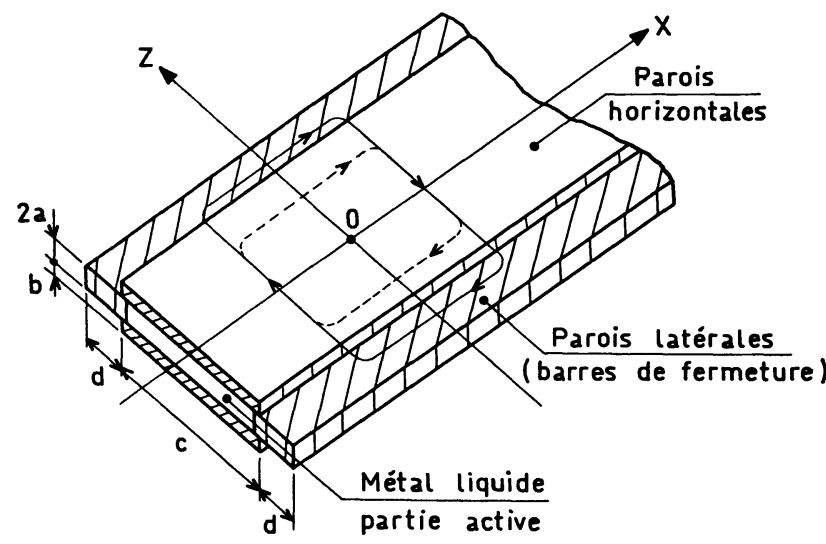

Fig. $2 b$.

Si nous considérons que le métal liquide est incompressible et se déplace, en pratique, comme un bloc solide, à une vitesse constante, nous pouvons l'assimiler, tout comme la bande conductrice du moteur linéaire située dans le champ magnétique à la partie active (droite) des bobines rotoriques d'une machine tournante classique. De la même façon les deux parties conductrices de largeur $d$, jouant le rôle de circuit de fermeture des courants induits dans la partie active (d'où leur appellation: " barres de fermeture "), peuvent être assimilées aux têtes de bobines de la machine tournante.

En ce qui concerne les parois horizontales du conduit M. H. D., il faut distinguer deux cas : si elles sont isolantes, elles se comportent comme une couche d'air intermédiaire entre l'induit et l'inducteur (couche 2 sur la figure 1) et les configurations transversales d'un moteur linéaire et d'une machine $M$. H. D. sont identiques; si elles sont conductrices (conduit en acier inoxydable par exemple), on doit les considérer comme un " induit " supplémentaire en court-circuit (car immobile par rapport au champ d'excitation), disposé en parallèle sur la partie conductrice active.

3. Equations générales du champ magnétique. Le champ magnétique constitue le paramètre essentiel qui distingue la machine asynchrone tournante de la machine linéaire dont la conception technologique lui est subordonnée.

La théorie de la machine linéaire a été établie à ses débuts à partir de celle des machines tournantes, cette dernière se développant à l'aide de modèles physiques approchés, dans lesquels la simplicité et la rapidité des calculs l'emportent très souvent sur l'exactitude mathématique du problème. Au point de vue électrodynamique, on peut intégrer la théorie des machines tournantes dans celle des circuits linéaires où les courants sont rigidement liés au système de conducteurs qui les véhiculent dans des directions bien déterminées; on suppose généralement que ces conducteurs sont filamentaires, c'est-à-dire de section nulle.

Or, si la méthode des circuits est applicable à l'inducteur de la machine linéaire, il n'en est pas de même pour l'induit où il convient de déterminer les variations spatiales et temporelles de la grandeur et de la direction du courant. Pour étudier les phénomènes physiques et magnétiques dans l'entrefer, il est donc nécessaire de faire appel à la théorie du champ électromagnétique ; c'est ainsi que deux des équations de Maxwell et la loi d'Ohm s'écrivent, avec les notations classiques, et pour un système de coordonnées liées aux inducteurs de la machine :

$$
\begin{aligned}
\operatorname{rot} \mathbf{B} & =\mu\left(\frac{\partial \mathbf{D}}{\partial t}+\frac{\mathbf{J}_{\mathbf{c}}}{2(a+b)}+\mathbf{J}\right) \\
\operatorname{rot} \mathbf{E} & =-\frac{\partial \mathbf{B}}{\partial t} \\
\mathbf{J} & =\frac{1}{\rho}(\mathbf{E}+\mathbf{V} \wedge \mathbf{B}) .
\end{aligned}
$$

Remarquons que l'équation de Maxwell réelle correspondant à (3) avec $\mu=$ cte serait :

$$
\operatorname{rot} \mathbf{B}=\mu\left[\frac{\partial \mathbf{D}}{\partial t}+\mathbf{J}\right]
$$

où $\mathbf{J}$ désigne la densité volumique des courants d'induit.

Lorsqu'on se propose de résoudre le système complet des équations avec ses conditions aux limites, on doit tenir compte de la nappe de courants filamentaires de densité linéiques $\mathbf{J}_{\mathbf{c}}$. Dans ce cas, les calculs sont malaisés; pour les résoudre plus facilement, nous sommes amenés à répartir dans la région 1 une densité de courant uniforme fictive supplémentaire

$$
\frac{J_{\mathrm{c}}}{2(a+b)}
$$

ainsi que nous l'avons explicité dans [18]. 
La constante de temps électriques des milieux conducteurs qui constituent l'induit de la machine étant très faible (de l'ordre de $10^{-17} \mathrm{~s}$ ), aucune densité de charges électriques ne peut s'y établir pratiquement ; d'où

$$
\frac{\partial \mathbf{D}}{\partial t}=\operatorname{div} \mathbf{J}=0 .
$$

En introduisant le potentiel vecteur $\mathbf{A}$, lié à $\mathbf{B}$ par

$$
\mathbf{B}=\operatorname{rot} \mathbf{A}
$$

et en appliquant le postulat de Lorentz, qui impose entre le potentiel vecteur et le potentiel scalaire $\psi$ du champ magnétique, les conditions particulières

$$
\operatorname{div} \mathbf{A}+\mu \varepsilon \frac{\partial \psi}{\partial t}=0
$$

nous obtenons, à partir de (3), (4), (5), (6), (7) et (8), l'expression

$$
\nabla^{2} \mathbf{A}-\frac{\mu}{\rho}\left(\frac{\partial \mathbf{A}}{\partial t}-\mathbf{V} \wedge \operatorname{rot} \mathbf{A}\right)=0
$$

qui détermine la distribution du champ magnétique. Les équations (4) et (5) donnent de plus la relation

$$
\operatorname{rot} \mathbf{J}=-\frac{1}{\rho}\left[\frac{\partial \mathbf{B}}{\partial t}+\operatorname{rot}(\mathbf{V} \wedge \mathbf{B})\right]
$$

qui régit la distribution des courants induits.

La résolution des équations (9) et (10) avec des conditions aux limites appropriées, qui permet théoriquement de déterminer tous les paramètres physiques de la machine linéaire à induction, est toutefois quasi impossible dans le cas général, à cause de la complexité des systèmes mathématiques qui interviennent. Afin de tirer des conclusions pratiques de ces équations, on peut, soit procéder à des simplifications parfois contestables, soit axer les études sur certains phénomènes isolés; l'image d'ensemble est alors obtenue par recoupement des différentes solutions. Nous avons choisi cette dernière voie en exposant séparément les études concernant d'une part les effets de pénétration du champ dans l'induit et d'autre part les effets d'extrémités.

4. Effet de pénétration du champ dans un induit linéaire. - Nous étudions les phénomènes liés à la distribution transversale du champ magnétique, c'està-dire l'effet de pénétration du champ à l'état propre (non perturbé par les effets d'extrémités), en supposant que ce champ est distribué le long de la machine sous forme d'une onde purement glissante, ce qui est seulement valable pour un dispositif infiniment long, muni de barres de fermeture de résistance négligeable.

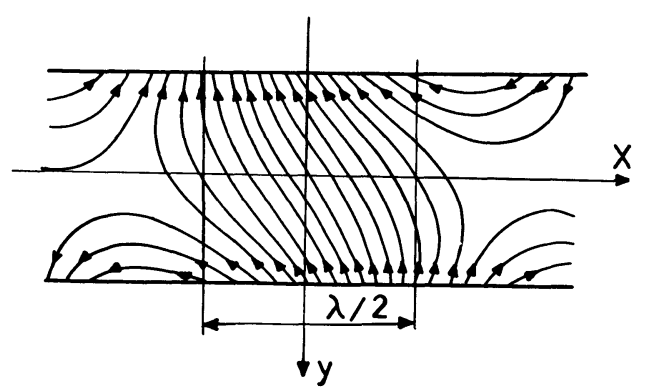

FIG. 3.

En désignant par $k=2 \pi \lambda^{-1}$, le numéro d'onde, nous posons dans la relation (1)

$$
\bar{f}(x)=\mathrm{e}^{-j k x}
$$

obtenant ainsi une distribution des courants d'excitation sous forme d'une onde sinusoïdale se propageant le long des inducteurs à la vitesse du champ

$$
V_{\mathrm{c}}=\frac{\mathrm{d} x}{\mathrm{~d} t}=\frac{\omega}{k} .
$$

Le potentiel vecteur s'écrit

$$
\mathbf{A}(x, y, t)=\operatorname{Re}\left\{\mathbf{u}_{z} \bar{A}(y) \mathrm{e}^{j(\omega t-k x)}\right\} .
$$

En affectant de l'indice $i=1$ ou 2 les grandeurs correspondant aux couches 1 et 2 de l'entrefer (Fig. 1), l'équation (9) se met sous la forme complexe :

$$
\frac{\partial^{2} \bar{A}_{\mathrm{i}}}{\partial y^{2}}-k^{2} \alpha_{\mathrm{i}}^{2} \bar{A}_{\mathrm{i}}=0
$$

avec

$$
\alpha_{\mathrm{i}}^{2}=1-j g R_{\mathrm{mi}}
$$

$g$ - glissement

$R_{\mathrm{mi}}=\frac{\mu_{\mathrm{i}} V_{\mathrm{c}}}{k \rho_{\mathrm{i}}}-$ nombre de Reynolds magnétique .

Pour simplifier les calculs nous éliminons les couches $2^{\prime}$ (isolation) et 3 (inducteurs) de l'étude en cours. La suppression de l'isolation entre l'inducteur et l'induit ne peut avoir qu'une influence quantitative que l'on peut d'ailleurs évaluer facilement par un calcul numérique approprié ; l'élimination des inducteurs revient à les considérer pratiquement comme des équipotentielles magnétiques en supposant

$$
\mu_{3} \gg \mu_{1} \text { et } \mu_{2} \text {. }
$$

Cette simplification nous prive ainsi de toute possibilité d'étude des phénomènes magnétiques dans les stators, mais une telle étude serait de toute façon entachée d'erreurs par suite de la représentation équivalente adoptée pour les courants d'excitation.

La solution générale de l'équation (11) s'écrit:

$$
\bar{A}_{\mathrm{i}}(x, y, t)=\left[\bar{A}_{\mathrm{i}}^{\prime} \mathrm{ch} . k \alpha_{\mathrm{i}} y+\bar{A}_{\mathrm{i}}^{\prime \prime} \operatorname{sh} . k \alpha_{\mathrm{i}} y\right] .
$$


Les quatre constantes d'intégration du système (12) se calculent à partir des conditions aux limites suivantes :

- à la limite entre les enroulements d'excitation et la couche 2

$$
\bar{B}_{x_{2}}=-\mu J_{c} \mathrm{e}^{j(\omega t-k x)} ;
$$

- à la limite des deux couches 1 et 2 de l'induit

$$
\frac{\bar{B}_{x_{1}}}{\mu_{1}}=\frac{\bar{B}_{x_{2}}}{\mu_{2}} \quad \text { et } \quad \bar{B}_{y_{1}}=\bar{B}_{y_{2}}
$$

- sur l'axe $O x$

$$
\bar{B}_{x_{1}}=0 \text {. }
$$

Nous obtenons, après quelques calculs [8]

$$
\left\{\begin{array}{r}
\mathbf{A}_{1}=\operatorname{Re} \mathbf{u}_{z}\left[\frac{\bar{K}_{1} \operatorname{ch} . k \alpha_{2} a+\operatorname{sh} . k \alpha_{2} a}{\operatorname{ch} . k \alpha_{2} a}\right] \times \bar{K}_{2} \operatorname{ch} . k \alpha_{1} y \cdot \mathrm{e}^{j(\omega t-k x)} \\
\quad \\
\mathbf{A}_{2}=\operatorname{Re} \mathbf{u}_{z}\left[\bar{K}_{1} \operatorname{ch} . k \alpha_{2} y+\operatorname{sh} . k \alpha_{2} y\right] \times \bar{K}_{2} \mathrm{e}^{j(\omega t-k x)}
\end{array}\right.
$$

avec

$$
\begin{aligned}
& \bar{K}_{1}= \frac{1-\frac{\alpha_{1} \mu_{2}}{\alpha_{2} \mu_{1}} \text { th. } k \alpha_{2} a \cdot \text { th. } k \alpha_{1} a}{\frac{\alpha_{1} \mu_{2}}{\alpha_{2} \mu_{1}} \operatorname{th} k \alpha_{1} a-\operatorname{th} k \alpha_{2} a} \\
& \bar{K}_{2}=-\frac{\mu_{2} J_{c}}{k \alpha_{2}} \times \\
& \quad \times\left[\frac{1}{\bar{K}_{1} \operatorname{sh} . k \alpha_{2}(a+b)+\operatorname{ch} \cdot k \alpha_{2}(a+b)}\right] .
\end{aligned}
$$

Notons que, dans un moteur linéaire et dans une machine M. H. D. à parois horizontales isolantes, où le nombre de Reynolds magnétique $R_{\mathrm{m}_{2}}$ est nul, on a

$$
\alpha_{2}=1 \text {. }
$$

Les composantes de l'induction $\mathbf{B}_{i}$

$$
\left(\frac{\partial \mathbf{A}_{\mathbf{i}}}{\partial y} ;-\frac{\partial \mathbf{A}_{\mathbf{i}}}{\partial x} ; 0\right)
$$

sont calculées à partir de (13) et la solution de l'équation :

$$
\frac{\mathrm{d} y}{\mathrm{~d} x}=-\frac{\operatorname{Re}\left\{\frac{\partial \bar{A}_{\mathrm{i}}}{\partial x}\right\}}{\operatorname{Re}\left\{\frac{\partial \bar{A}_{\mathrm{i}}}{\partial y}\right\}}
$$

permet de tracer les lignes d'induction dans l'entrefer. La figure 3 indique les résultats de la programmation du système (14) pour une machine de caractéristiques :

$$
2(a+b)=2 \times 10^{-2} \mathrm{~m}, \quad R_{\mathrm{m}_{1}}=50 .
$$

Remarquons que des tracés semblables ont été dérivés par ailleurs de systèmes simplifiés [7].

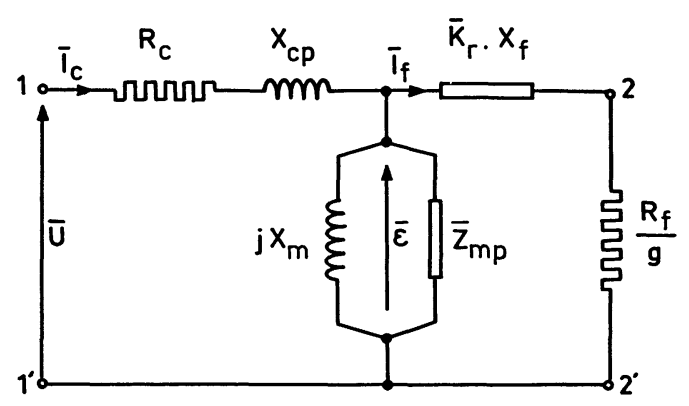

FIG. 4.

La déformation du champ d'induction transversal, qui provient de sa diffusion dans un conducteur en mouvement, se traduit par un infléchissement des lignes de forces ; le champ ne peut plus être considéré comme plan parallèle et la diminution de sa composante utile $B_{y}$ est d'autant plus importante que le facteur

$$
\text { ch. } k \alpha_{1} y=\operatorname{ch} .2 \pi \alpha_{1} \frac{y}{\lambda}
$$

est plus grand ; cette diminution est donc négligeable pour les machines dont l'entrefer est petit devant le pas polaire. On définit l'épaisseur critique $a_{0}$ de l'entrefer, correspondant à une réduction de $B_{y}$ dans le rapport $e$; cette profondeur de pénétration, analogue à l'effet de peau dans un conducteur, est donnée, pour une machine où l'épaisseur $b$ de la partie 2 est très faible, par une relation de la forme

$$
a_{0}=\frac{K_{0}}{K_{01}+g R_{\mathrm{m}_{1}}} .
$$

N'insistons pas sur l'expression, complexe, des coefficients $K_{0}$ et $K_{01}$, obtenus par ailleurs [8]. Signalons simplement que, pour une machine à un seul inducteur, on retrouve la formule

$$
a_{0}=\lambda \sqrt{\frac{1}{2 \pi^{2} \sqrt{1+\left(g R_{\mathrm{m}_{1}}\right)^{2}}+1}}
$$

donnée par d'autres auteurs [3], [9].

La déformation des lignes d'induction influe sur le couplage magnétique entre l'inducteur et l'induit, et provoque une augmentation des pertes magnétiques et ohmiques de l'induit.

Il est possible de tenir compte des effets de pénétration sur le schéma électrique équivalent dont nous avons développé la théorie par ailleurs [10], [11]. Ce schéma (Fig. 4) ne diffère de celui de la machine tournante que par les deux coefficients $\bar{Z}_{\text {mp }}$ et $\bar{K}_{\mathrm{r}}$ affectés respectivement au couplage magnétique et à l'impédance de fuites de l'induit, et dont l'étude permet d'évaluer les effets de pénétration.

L'influence des coefficients $\bar{Z}_{\mathrm{mp}}$ et $\bar{K}_{\mathrm{r}}$ est pratiquement négligeable pour

$$
k a g R_{\mathrm{mi}} \ll 1 .
$$


On peut donc considérer comme plan parallèle le champ magnétique dans l'entrefer d'une machine linéaire à condition que son pas polaire soit grand devant la quantité $a g R_{\mathrm{m}}$, qui apparaît ainsi clairement comme un " entrefer équivalent ».

S'il est illusoire de tirer du schéma précédent des conclusions énergétiques valables pour une machine réelle dans laquelle le champ n'a pas une distribution longitudinale purement glissante, nous pouvons par contre en déduire des renseignements concernant les phénomènes liés au couplage magnétique; par exemple, le facteur de puissance de la machine s'écrit, en désignant par $\bar{Z}$ l'impédance totale vue des bornes d'entrée du quadripôle.

$$
\cos \varphi=\frac{\operatorname{Re}\{\bar{Z}\}}{Z}=\frac{g R_{\mathrm{m}_{1}}}{\sqrt{1+g^{2} R_{\mathrm{m}_{1}}^{2}}} \cdot K_{\varphi}
$$

où

$$
K_{\varphi}=\left[\left(1+\frac{X_{\mathrm{cp}}}{X_{\mathrm{m}}}\right)^{2}+\left(\frac{X_{\mathrm{cp}}}{X_{\mathrm{m}}} g R_{\mathrm{m}_{1}}\right)\right]^{-1 / 2} \leqslant 1 .
$$

En négligeant la réactance de fuites du stator $X_{\mathrm{cp}}$ devant celle du couplage magnétique $X_{\mathrm{m}}$, nous retrouvons l'expression du facteur de puissance donnée par de nombreux auteurs [12], [13].

5. Effets d'extrémités. - 5.1 DéfInITION. - Le circuit magnétique, plan et limité dans l'espace, ne peut créer dans l'entrefer, un champ purement glissant équivalent au champ tournant des machines classiques. L'expression du champ résultant approché des machines linéaires, que nous avons étudiée plus haut, est perturbée par un ensemble de phénomènes appelés "effets d'extrémités ", divisés généralement, quoique assez arbitrairement, en deux parties :

l'effet de longueur finie, qui est dû principalement — à la variation de perméabilité à l'entrée et à la sortie,

- aux flux parasites se fermant aux extrémités et sur les côtés,

- aux f. é. m. à l'entrée et à la sortie dues aux courants d'induit, qui se ferment en dehors de la carcasse magnétique de la machine ;

- aux f. é. m. dues à la discontinuité de l'induction à l'entrée et à la sortie ;

l'effet de largeur finie, dû à la fermeture des courants à l'intérieur de la partie active de l'induit.

5.2 EFFET DE LONGUEUR FINIE. - Nous l'évaluerons en considérant le modèle de la figure 5 que nous avons initialement proposé [14], [15] et qui a été utilisé par d'autres auteurs [16], [17]. Ce modèle comporte cinq zones d'axe $O x$ :

- la zone utile III, de longueur $p \lambda$, à laquelle correspond la distribution linéique de courant inducteur $\mathbf{J}_{c}$, engendrant le champ d'induction unidimen-

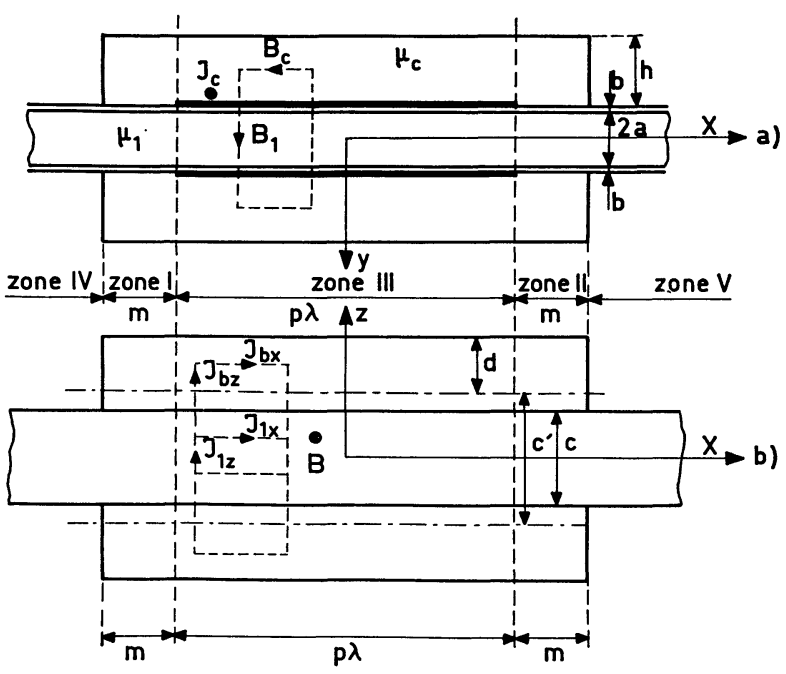

FIG. 5.

sionnel $\mathbf{B}(O, B, O)$, supposé plan parallèle et implique la relation (16) satisfaite,

- les zones I et II, de longueur $m$, complétant le circuit magnétique et symbolisant le flux de fuites,

- les zones IV et V, illimitées, prolongeant le circuit d'induit et dépourvues de tout couplage magnétique avec les précédentes.

Dans une machine réelle, où une partie de barres de fermeture peut se trouver dans le champ magnétique, il convient de tenir compte des fuites magnétiques latérales (points $z= \pm c / 2$ ); nous sommes ainsi conduits à définir, comme nous l'expliquerons plus loin, en désignant par $c^{\prime}-c$ l'épaisseur de pénétration du champ dans les barres, une résistivité équivalente

$$
\rho_{1 b}=\rho_{1}+\rho_{b}\left(\frac{c^{\prime}-c}{c}\right)
$$

avec

$\rho_{b}$ - résistivité des barres de fermeture.

En supposant $\mu_{1}=\mu_{2}$ et $\rho_{b} \ll \rho_{1}$, ce qui revient à négliger l'effet de largeur, nous obtenons à partir des expressions (3) et (10), un système d'équations, déterminant dans chaque zone de la figure 5, la distribution du flux magnétique et des courants induits, qui, dans les barres de fermeture, sont affectés de l'indice $b$.

$$
\begin{aligned}
& \nabla^{2} \bar{\varphi}_{\mathrm{III}}-\frac{\mu_{1}}{\mu_{3}(a+b) h} \bar{\varphi}_{\mathrm{III}}=\frac{\mu_{1}}{2(a+b)} \bar{J}_{\mathrm{c}} \mathrm{e}^{j(\omega t-k x)}+ \\
& +\frac{a \mu_{1} d}{a+b} \frac{\partial \vec{J}_{b x I I I}}{\partial x} \\
& \nabla^{2} \bar{J}_{b x \mathrm{III}}-\frac{2 \rho_{b}}{\rho_{1 b} c d} \bar{J}_{b x \mathrm{III}}=\frac{V}{\rho_{1 b} d} \nabla^{2} \bar{\varphi}_{\mathrm{III}}+ \\
& +\frac{c^{\prime}}{c} \frac{j \omega}{\rho_{1 b} d} \frac{\partial \bar{\varphi}}{\partial x} \\
& \nabla^{2} \bar{\varphi}_{\mathrm{I}, \mathrm{II}}-\frac{\mu_{1}}{\mu_{3}(a+b) h} \bar{\varphi}_{\mathrm{I}, \mathrm{II}}=\frac{a \mu_{1} d}{(a+b)} \frac{\partial \bar{J}_{b x, \mathrm{I}, \mathrm{II}}}{\partial x}
\end{aligned}
$$




$$
\begin{gathered}
\nabla^{2} \bar{J}_{b x, \mathrm{IIII}}-\frac{2 \rho_{b}}{\rho_{1 b} d c} \bar{J}_{b x \mathrm{I}, \mathrm{II}}=\frac{V}{\rho_{1 b} d} \nabla^{2} \bar{\varphi}_{\mathrm{I}, \mathrm{II}}+ \\
+\frac{c^{\prime}}{c} \frac{j \omega}{\rho_{1 b} d} \frac{\partial \bar{\varphi}_{\mathrm{I}, \mathrm{II}}}{\partial x} \\
\nabla^{2} \bar{J}_{1 x \mathrm{IV}, \mathrm{V}}-\frac{2}{\left(\frac{c-c^{\prime \prime}}{2}\right) c^{\prime \prime}} \bar{J}_{1 x \mathrm{IV}, \mathrm{v}}=0
\end{gathered}
$$

où' $c^{\prime \prime}$.(cf.'[14]) désigne la longueur de la partie « droite » (selon $\mathrm{Oz}$ ) des courants dans les zones d'extrémités.

La relation

$$
\operatorname{div} \bar{J}_{1}=0
$$

donne

$$
\bar{J}_{1 z}=\frac{\partial \bar{J}_{b x}}{\partial x} \cdot d
$$

La solution générale des équations (17), (18) et (19) s'écrit

$$
\begin{aligned}
& \left\{\begin{array}{l}
\bar{\varphi}_{\mathrm{III}}=\bar{\varphi}_{0} \mathrm{e}^{-j k x}+\sum_{n=1}^{4} \bar{C}_{n} \mathrm{e}^{\bar{r}_{n} x} \\
\bar{J}_{b \mathrm{III}}=\bar{J}_{0} \mathrm{e}^{-j k x}+\sum_{n=1}^{4} \bar{K}_{n} \bar{C}_{n} \mathrm{e}^{\bar{r}_{n} x}
\end{array}\right. \\
& \left\{\begin{array}{l}
\bar{\varphi}_{\mathrm{I}}=\sum_{n=1}^{4} \bar{C}_{n+4} \mathrm{e}^{\bar{r}_{n} x} \\
\bar{J}_{b \mathrm{I}}=\sum_{n=1}^{4} \bar{K}_{n} \bar{C}_{n+4} \mathrm{e}^{\bar{r}_{n} x} \\
\bar{\varphi}_{\mathrm{II}}=\sum_{n=1}^{4} \bar{C}_{n+8} \mathrm{e}^{\bar{r}_{n} x} \\
\bar{J}_{b \mathrm{II}}=\sum_{n=1}^{4} \bar{K}_{n} \bar{C}_{n+8} \mathrm{e}^{\bar{r}_{n} x}
\end{array}\right. \\
& \bar{J}_{1 x \mathrm{IV}}=\bar{C}_{13} \mathrm{e}^{r_{5} x} ; \quad \bar{J}_{1 x \mathrm{~V}}=\bar{C}_{14} \mathrm{e}^{-r_{5} x} .
\end{aligned}
$$

Les constantes d'intégration des expressions (20) à (23) sont calculées à partir des conditions aux limites suivantes :

$$
\begin{aligned}
& \text { - pour } \\
& \qquad=-\left(m+p \frac{\lambda}{2}\right) \pm \varepsilon \\
& \bar{\varphi}_{\mathrm{I}}=0 ; \bar{J}_{1 z \mathrm{IV}}=\bar{J}_{1 z \mathrm{I}} ; \\
& \quad \frac{\partial \bar{J}_{1 z \mathrm{IV}}}{\partial x} \cdot \rho_{1} c^{\prime \prime} \frac{c-c^{\prime \prime}}{2}-\frac{\partial \bar{J}_{1 z \mathrm{I}}}{\partial x} \cdot \rho_{1} c d=V \cdot c \frac{\partial \bar{\varphi}_{\mathrm{I}}}{\partial x} \\
& - \text { pour }
\end{aligned}
$$

$$
x=\left(m+p \frac{\lambda}{2}\right) \pm \varepsilon
$$

$$
\begin{aligned}
\bar{\varphi}_{\mathrm{II}}=0 ; \bar{J}_{1 \mathrm{zV}} & =\bar{J}_{1 z \mathrm{II}} ; \\
& \frac{\partial \bar{J}_{1 z \mathrm{~V}}}{\partial x} \cdot \rho_{1} c^{\prime \prime} \frac{c-c^{\prime \prime}}{2}-\frac{\partial \bar{J}_{1 \mathrm{III}}}{\partial x}=V c \frac{\partial \bar{\varphi}_{\mathrm{II}}}{\partial x} ;
\end{aligned}
$$

- pour

$$
\begin{gathered}
x=-p \frac{\lambda}{2} \\
\bar{\varphi}_{\mathrm{I}}=\bar{\varphi}_{\mathrm{III}} ; \bar{J}_{1 z \mathrm{I}}=\bar{J}_{1 z \mathrm{III}} ; \\
\frac{\partial \bar{\varphi}_{\mathrm{I}}}{\partial x}=\frac{\partial \bar{\varphi}_{\mathrm{III}}}{\partial x} ; \frac{\partial \bar{J}_{1 z \mathrm{I}}}{\partial x}=\frac{\partial \bar{J}_{1 z \mathrm{III}}}{\partial x}
\end{gathered}
$$$$
\text { - pour }
$$

$$
x=p \frac{\lambda}{2}
$$

$\bar{\varphi}_{\mathrm{II}}=\bar{\varphi}_{\mathrm{III}} ; \bar{J}_{1 z \mathrm{II}}=\bar{J}_{1 z \mathrm{III}} ;$

$$
\frac{\partial \bar{\varphi}_{\text {II }}}{\partial x}=\frac{\partial \bar{\varphi}_{\text {III }}}{\partial x} ; \frac{\partial \bar{J}_{1 z \text { II }}}{\partial x}=\frac{\partial \bar{J}_{1 z \text { III }}}{\partial x} ;
$$

- pour $x \rightarrow-\infty$

$$
\bar{J}_{1 \mathrm{IV}} \rightarrow 0
$$

- pour $x \rightarrow \infty$

$$
\bar{J}_{1 \mathrm{v}} \rightarrow 0 \text {. }
$$

Les exponentielles $r_{n}$ se déduisent du wronskien du système (17) tandis que les termes $\bar{\varphi}_{0}, \bar{J}_{0}$ et $\bar{K}_{n}$ s'écrivent, après quelques calculs

$$
\bar{J}_{0}=\bar{\varphi}_{0} \frac{k\left(V c k+\omega c^{\prime}\right)}{2 \rho_{b}+\rho_{1 b} d c k^{2}}
$$

$$
\begin{aligned}
& \bar{\varphi}_{0}= \\
& \begin{aligned}
-\frac{\bar{J}_{c} \mu_{1}}{2(a+b)}\left[k^{2}+\right. & \frac{\mu_{1}}{\mu_{3}(a+b) h}+ \\
& \left.+j \frac{a k^{2} \mu_{1} d\left(k V c+\omega c^{\prime}\right)}{(a+b)\left(k^{2} \rho_{1 b} d c+2 \rho_{b}\right)}\right] \mathrm{e}^{j \omega t}
\end{aligned}
\end{aligned}
$$

$$
\begin{gathered}
{\left[k^{2}+\frac{\mu_{1}}{\mu_{3}(a+b) h}\right]^{2}+} \\
+\left[\frac{a k^{2} \mu_{1} d\left(k V c+\omega c^{\prime}\right)}{(a+b)\left(k^{2} \rho_{1 b} d c+2 \rho_{b}\right)}\right]^{2} \\
\bar{K}_{n}=\frac{\bar{r}_{n}^{2} \mu_{3}(a+b) h-\mu_{1}}{\mu_{1} \mu_{3} a d h r_{n}^{2}} .
\end{gathered}
$$

Pour une machine à vide $\left(J_{1}=0\right)$, la solution (20) se simplifie considérablement; nous obtenons

$$
\bar{\varphi}_{\text {III }}=\bar{\varphi}_{0} \mathrm{e}^{-j k x}+\bar{C}_{1} \mathrm{e}^{r_{0} x}+C_{2} \mathrm{e}^{-r_{0} x}
$$

avec

$$
r_{0}=\sqrt{\frac{\mu_{1}}{\mu_{3} h(a+b)}}
$$




$$
\begin{aligned}
& \bar{\varphi}_{0}=- \frac{\mu_{1} J_{c}}{2(a+b)\left(k^{2}+r_{0}^{2}\right)} \mathrm{e}^{j \omega t} \\
& \bar{C}_{1}=- \varphi_{0}\left\{\frac{j k}{r_{0}} \operatorname{sh} r_{0} m \cdot \operatorname{ch}\left[p \frac{\lambda}{2}\left(j k-r_{0}\right)-m r_{0}\right]+\right. \\
&\left.+\operatorname{ch} r_{0} m \cdot \operatorname{sh}\left[p \frac{\lambda}{2}\left(j k-r_{0}\right)-m r_{0}\right]\right\} \times \\
& \times\left\{\operatorname{sh}\left[r_{0}(p \lambda+2 m)\right]\right\}^{-1} \\
& \bar{C}_{2}=\bar{\varphi}_{0}\left\{\frac{j k}{r_{0}} \operatorname{sh} r_{0} m \cdot \operatorname{ch}\left[p \frac{\lambda}{2}\left(j k+r_{0}\right)+m r_{0}\right]+\right. \\
&\left.+\operatorname{ch~} r_{0} m \cdot \operatorname{sh}\left[p \frac{\lambda}{2}\left(j k+r_{0}\right)+m r_{0}\right]\right\} \times \\
& \times\left\{\operatorname{sh}\left[r_{0}(p \lambda+2 m)\right]\right\}^{-1} .(25 b)
\end{aligned}
$$

Signalons que l'expression ci-dessus peut être mise sous une forme semblable à celle obtenue par Shturman [1] pour une machine à nombre pair de pôles.

D'une manière générale, nous remarquons, à partir des expressions (20) et (24), que l'effet de longueur finie se caractérise par l'apparition dans l'entrefer, à côté du champ purement glissant, de composantes pulsatoires qui se propagent le long de l'inducteur avec des amplitudes variables. En charge, cet effet est responsable de la distribution spécifique des courants induits qui sortent aux extrémités de la machine.

Une analyse plus approfondie des équations (20) et (24), seulement possible par calcul numérique, a été développée dans [18].

Les figures $6 a$ et $6 b$ représentent la distorsion du champ magnétique à vide en fonction de la longueur $2 m$ des zones magnétiques non bobinées, rapportée à la longueur d'onde. On constate aisément que, suivant



Fig. $6 u$. que la machine est à nombre pair ou impair de pôles la distorsion est une fonction croissante ou décroissante de $m$. Les courbes programmées ont été confrontées avec les mesures obtenues sur le moteur linéaire de la figure 12 .

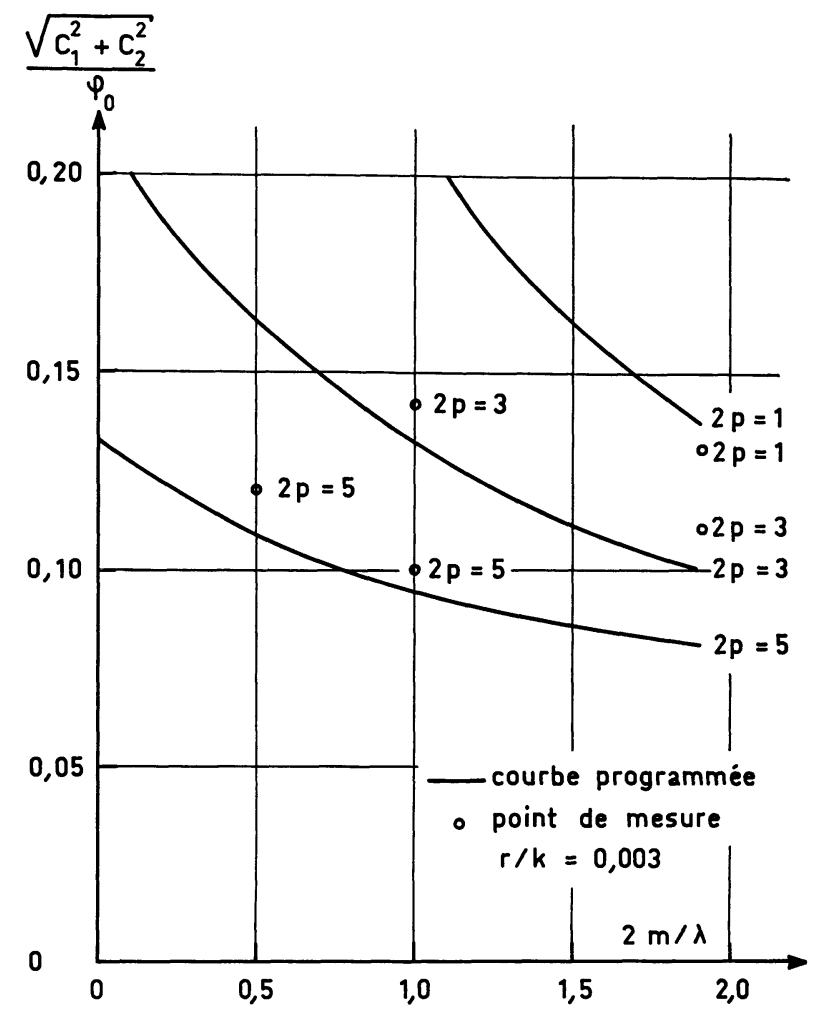

FIG. $6 b$.

5.3 Effet de LARgeUr Finie. - Le champ d'induction, créé par les courants d'excitation de direction $O z$, comporte à la fois une onde principale glissante et des ondes parasites asynchrones, c'est-à-dire liées au référentiel de l'induit. L'onde principale engendre une onde glissante de $f$. é. m., qui change de signe sur un pas polaire, provoquant ainsi la fermeture des courants induits correspondants. Si la majeure partie de ces courants $\left(J_{b x}\right.$ sur la figure 5) se boucle dans les barres de fermeture situées en principe hors du champ, une fraction parasite $\left(J_{1 x}\right.$ sur la figure 5$)$ se ferme à l'intérieur de la partie active de l'induit. Ces courants parasites diminuent la composante utile $J_{1 z}$, donc la poussée de la machine et leur importance dépendent de la longueur relative des parties où agissent et s'annulent les f. é. m. utiles. Ainsi, l'effet de largeur finie est d'autant plus grand que la largeur de la partie active de l'induit est plus faible devant son pas polaire. Dans une machine infiniment large, cet effet disparaît complètement. Nous évaluons dans ce paragraphe son influence en calculant la distribution des courants induits dans une seule zone (zone III de la figure 5 limitée $\mathrm{par}-\mathrm{p} \lambda / 2<x<\mathrm{p} \lambda / 2)$, c'est-à-dire sans tenir compte de la longueur de la machine. Nous sup- 
posons que l'induction dans l'entrefer de cette zone est de la forme générale

$$
\bar{B}_{1 \mathrm{III}}=\bar{B}_{1}=f(\bar{z}) \sum_{n}\left(\alpha_{n}-j \beta_{n}\right) \mathrm{e}^{j\left(\omega t-k_{n} x\right)} .
$$

Cette induction, engendrée à la fois par les courants statoriques et par les courants induits, admet une asymétrie de sa distribution selon $O x$ (provoquée par exemple par un effet de longueur finie) et une variation en $z$ qui résulte de la réaction d'induit provoquée par les courants se fermant suivant $O x$. Nous avons montré [18] qu'il est souhaitable, au point de vue mathématique, de reporter cette dernière variation sur les courants d'excitation en admettant que

$$
f(\bar{z})=1
$$

à condition que

$$
\bar{J}_{c}=f(x, z, t) \text {. }
$$

A partir des expressions (6), (10) et sous réserve de la dérivabilité par terme de la relation (26), on obtient les relations suivantes

$$
\begin{aligned}
& \left\{\begin{array}{l}
\nabla^{2} \bar{J}_{1 z}=\sum_{n} \frac{k_{n} V_{c n}}{\rho_{1}} g_{n}\left(\alpha_{n}-j \beta_{n}\right) \mathrm{e}^{j\left(\omega t-k_{n} x\right)} \\
\nabla^{2} \bar{J}_{1 x}=0
\end{array}\right. \\
& \left\{\begin{array}{l}
\nabla^{2} \bar{J}_{b z}=0 \\
\nabla^{2} \bar{J}_{b x}=0
\end{array}\right.
\end{aligned}
$$

en introduisant les nouvelles valeurs, variables, du nombre d'onde $k_{n}=n k$, de la vitesse du champ

$$
V_{c n}=\omega k_{n}^{-1}
$$

et du glissement $g_{n}=1-V . V_{c n}^{-1}$.

La méthode de séparation des variables permet d'écrire la solution générale des systèmes (27) et (28) sous la forme

$$
\begin{aligned}
\bar{J}_{1 z}=\sum_{n}\left(\bar{A}_{1 n} \mathrm{e}^{j k_{n} x}+\bar{A}_{2 n} \mathrm{e}^{-j k_{n} x}+\bar{A}_{3 n}\right) \times \\
\times\left(\bar{B}_{1 n} \mathrm{e}^{k_{n} z}+\bar{B}_{2 n} \mathrm{e}^{-k_{n} z}+\bar{B}_{3 n}\right) \\
\bar{J}_{1 x}=\sum_{n}\left(\bar{A}_{1 n}^{\prime} \mathrm{e}^{j k_{n} x}+\bar{A}_{2 n}^{\prime} \mathrm{e}^{-j k_{n} x}\right) \times \\
\times\left(\bar{B}_{1 n}^{\prime} \mathrm{e}^{k_{n} z}+\bar{B}_{2 n}^{\prime} \mathrm{e}^{-k_{n} z}\right)
\end{aligned}
$$

$$
\begin{aligned}
\bar{J}_{b z}=\sum_{n}\left(\bar{C}_{1 n} \mathrm{e}^{j k_{n} x}+\bar{C}_{2 n} \mathrm{e}^{-j k_{n} x}\right) \times \\
\times\left(\bar{D}_{1 n} \mathrm{e}^{k_{n} z}+\bar{D}_{2 n} \mathrm{e}^{-k_{n} z}\right) \\
\bar{J}_{b x}=\sum_{n}\left(\bar{C}_{1 n}^{\prime} \mathrm{e}^{j k_{n} x}+\bar{C}_{2 n}^{\prime} \mathrm{e}^{-j k_{n} x}\right) \times \\
\times\left(\bar{D}_{1 n}^{\prime} \mathrm{e}^{k_{n} z}+\bar{D}_{2 n}^{\prime} \mathrm{e}^{-k_{n} z}\right) .
\end{aligned}
$$

En tenant compte de la symétrie axiale des systèmes (27) et (28) il suffit, pour déterminer toutes les constantes d'intégration des équations (25), des six conditions aux limites :
1) $\operatorname{div} \mathbf{J}_{\boldsymbol{b}}=0$,

2) $J_{b z}=0$ pour $z=c / 2+d$,

3) $\operatorname{div} \mathbf{J}_{1}=0$,

4) $J_{1 z}=J_{b z}$ pour $z=c / 2$,

5) $J_{1 x}=0$ pour $z=0$,

6) $J_{1 x} \rho_{1}=J_{b x} \rho_{b}$ pour $z=c / 2$.

Si les cinq premières conditions sont toujours remplies pour la configuration considérée, il n'en est pas de même de la sixième qui appelle quelques commentaires dans les deux cas à envisager :

- dans le moteur linéaire, les barres de fermeture se déplacent à la même vitesse que la partie active de l'induit ; l'égalité des f. é. m. tangentielles n'est en principe valable qu'écrite dans un système de coordonnées mobiles, liées, par exemple à l'induit ;

- dans une machine M. H. D., les barres de fermeture sont immobiles et la vitesse subit une discontinuité en passant de la valeur $V$ dans la partie active de l'induit (métal liquide) à 0 ; l'égalité des f. é. $m$. tangentielles n'est valable [19] que si les champs électriques dans les deux régions sont définis sur un référentiel unique, lié par exemple à l'inducteur.

Cependant, nous avons montré [18] que, pour des systèmes non relativistes, les deux versions de la sixième condition donnent un résultat identique si l'on considère qu'aucun flux ne pénètre dans les barres de fermeture.

Le calcul, relativement complexe, permet, en posant

$$
\begin{gathered}
J_{n}=\frac{V_{c n} g_{n}}{\rho_{1}} \\
K_{n_{1}}=\frac{1}{\frac{\rho_{1}}{\rho_{b}} \operatorname{sh} \cdot k_{n} \frac{c}{2} \operatorname{th} k_{n} d+\operatorname{ch} k_{n} \frac{c}{2}} \\
\frac{c}{2} \leqslant z_{1} \leqslant \frac{c}{2}+d \quad 0 \leqslant z \leqslant \frac{c}{2}
\end{gathered}
$$

d'obtenir la solution particulière des systèmes (27) et (28)

$$
\begin{gathered}
\bar{J}_{1 z}=\sum_{n}\left(\alpha_{n}-j \beta_{n}\right) J_{n}\left[K_{n_{1}} \operatorname{ch} k_{n} z-1\right] \mathrm{e}^{j\left(\omega t-k_{n} x\right)} \\
\bar{J}_{1 x}=-j \sum_{n}\left(\alpha_{n}-j \beta_{n}\right) J_{n} K_{n_{1}} \operatorname{sh} k_{n} z \cdot \mathrm{e}^{j\left(\omega t-k_{n} x\right)} \\
\bar{J}_{b x}=+j \sum_{n}\left(\alpha_{n}-j \beta_{n}\right) \frac{J_{n}}{\operatorname{sh} k_{n} d}\left[K_{n_{1}} \operatorname{ch} k_{n} \frac{c}{2}-1\right] \times \\
\times \operatorname{ch}\left[k_{n}\left(z_{1}-\frac{c}{2}-d\right)\right] \mathrm{e}^{j\left(\omega t-k_{n} x\right)} \\
\bar{J}_{b z}=-\sum_{n}\left(\alpha_{n}-j \beta_{n}\right) \frac{J_{n}}{\operatorname{sh} k_{n} d}\left[K_{n_{1}} \operatorname{ch} k_{n} \frac{c}{2}-1\right] \times \\
\times \operatorname{sh}\left[k_{n}\left(z_{1}-\frac{c}{2}-d\right)\right] \mathrm{e}^{j\left(\omega t-k_{n} x\right)} .
\end{gathered}
$$

Il est important de remarquer que, selon (32), le courant $J_{1 x}$ et, partant, l'effet de largeur, sont négli- 
geables lorsque $K_{n 1}$ tend vers zéro, ce qui est réalisé, d'après (31) :

soit pour $k_{n} c / 2 \rightarrow \infty$, valable pour une machine infiniment large,

soit pour $\rho_{1} \gg \rho_{b}$, correspondant par exemple à des barres de fermeture supraconductrices.

Pour obtenir la distribution des courants induits dans une machine infiniment longue, où l'induction résultante (26) se réduit au seul terme fondamental, il convient évidemment de poser $n=1$ dans les expressions (31) et (32).

5.4 Considération Simultanée Des EFFETS DE LARGEUR ET DE LONGUEUR FINIES. - On a démontré [16], [17], [18] la validité d'utilisation du modèle magnétique de la figure 5 pour l'étude d'ensemble des effets d'extrémités. Bornons-nous à indiquer, par souci de simplification, l'exemple de son application à une machine sans barres de fermeture et où, par conséquent, les effets parasites sont particulièrement amplifiés ; signalons cependant que l'étude a été faite par ailleurs [18] dans le cas général. En considérant l'inducteur comme magnétiquement équipotentiel, nous pouvons, compte tenu de la relation (6) grouper les systèmes (17) et (18) en une seule équation. Nous obtenons ainsi, dans la zone III

$$
\begin{aligned}
\nabla^{2} \bar{J}_{1 z \text { III }}+k R_{m_{1}}\left[(g-1) \frac{\partial \bar{J}_{1 z \text { III }}}{\partial x}\right. & \left.-j k \bar{J}_{1 z \text { IIII }}\right]+ \\
& -j k^{2} R_{m_{1}} \bar{J}_{c}=0
\end{aligned}
$$

où

$$
\bar{J}_{c}=\bar{J}_{c} \mathrm{e}^{j(\omega t-k x)} .
$$

L'équation (33) se réduit, dans les zones I et II aux deux premiers termes et, dans les zones IV et $\mathrm{V}$ au seul premier terme. En tenant compte des conditions de symétrie 2) et 5) du système (30), la solution générale de l'équation (33) prend la forme d'une série géométrique paire $q(i)$

$$
\begin{array}{r}
\bar{J}_{1 z \mathrm{III}}=\sum_{i}\left[\bar{K}_{i \mathrm{III} 1} \cdot \exp \left[\left(s+\bar{r}_{i}\right)\left(x+p \frac{\lambda}{2}\right)\right]+\right. \\
\left.+\bar{K}_{i \mathrm{III} 2} \exp \left[\left(s-\bar{r}_{i}\right)\left(x+p \frac{\bar{\lambda}}{2}\right)\right]+\bar{K}_{o i} \exp -j k x\right] \times \\
\times \cos k_{q(i)} \cdot z
\end{array}
$$

avec

$$
k_{i}=k i \frac{\lambda}{2 c}
$$

et

$$
q(i)=\frac{i-1}{2}
$$

et en posant :

$$
s=\frac{k(1-g)}{2} R_{m_{1}}
$$

$$
\begin{aligned}
& \bar{r}_{i}=k\left[\left(\frac{1-g}{2}\right)^{2} R_{m_{1}}^{2}-j R_{m_{1}}+\left(\frac{\lambda(i-1)}{4 c}\right)^{2}\right]^{1 / 2} \\
& \bar{K}_{o i}=j(-1)^{i} i^{-3} \frac{g R_{m_{1}}}{k a \bar{K}_{i}} \\
& \bar{K}_{i}=2^{-4}\left[\frac{\lambda^{3}}{c^{3}}-\frac{4\left(\alpha_{1}-2\right)}{c} \lambda_{i}-2\right]
\end{aligned}
$$

$\alpha_{1}$ est donné par l'expression (11).

Connaissant la valeur de $J_{1 z \mathrm{IIII}}$, on peut déterminer à partir des relations (3) et (6), à une fonction paire $\bar{f}(z)$ près, les expressions $J_{1 z \mathrm{III}}$ et $B_{1 \mathrm{III}}$

$$
\begin{gathered}
\bar{B}_{1 \mathrm{III}}=\sum_{i}\left[\frac{\mu_{1}}{s+\bar{K}_{i \mathrm{III} 1}} \exp \left[\left(s+\bar{r}_{i}\right)\left(x-p \frac{\lambda}{2}\right)\right]+\right. \\
+\frac{\mu_{1} \bar{K}_{i \mathrm{III} 2}}{s-\bar{r}_{i}} \exp \left[\left(s-\bar{r}_{i}\right)\left(x+p \frac{\lambda}{2}\right)\right] \\
\left.+j \frac{\mu_{1} \bar{K}_{o i}}{k} \exp -j k x\right] \cos k_{q(i)} \cdot z+ \\
+j \frac{J_{c} \mu^{1}}{2 a k} \exp -j k x+\bar{f}(z)
\end{gathered}
$$

$$
\begin{aligned}
\bar{J}_{1 x \mathrm{III}}=\sum_{i} k_{q(i)}\left[\frac{\bar{K}_{i \mathrm{III} 1}}{s+\bar{r}_{i}} \exp \left[\left(s+\bar{r}_{i}\right)\left(x-p \frac{\lambda}{2}\right)\right]+\right. \\
+\frac{\bar{K}_{i \mathrm{III} 2}}{s-r_{i}} \exp \left[\left(s-\bar{r}_{i}\right)\left(x+p \frac{\lambda}{2}\right)\right]+ \\
\left.\quad+j-\bar{K}_{o i} \exp -j k x\right] \sin k_{q(i)} \cdot z+\frac{\partial \bar{f}(z)}{\partial z} .
\end{aligned}
$$

Les valeurs de l'induction et des courants dans les autres zones se déduisent aisément des formules (34) (35) et (36). L'exploration numérique des résultats obtenus est possible à l'aide de huit des douze conditions aux limites du paragraphe 5.2.

La figure 7 compare la distribution de la valeur efficace de l'induction le long de la machine, calculée

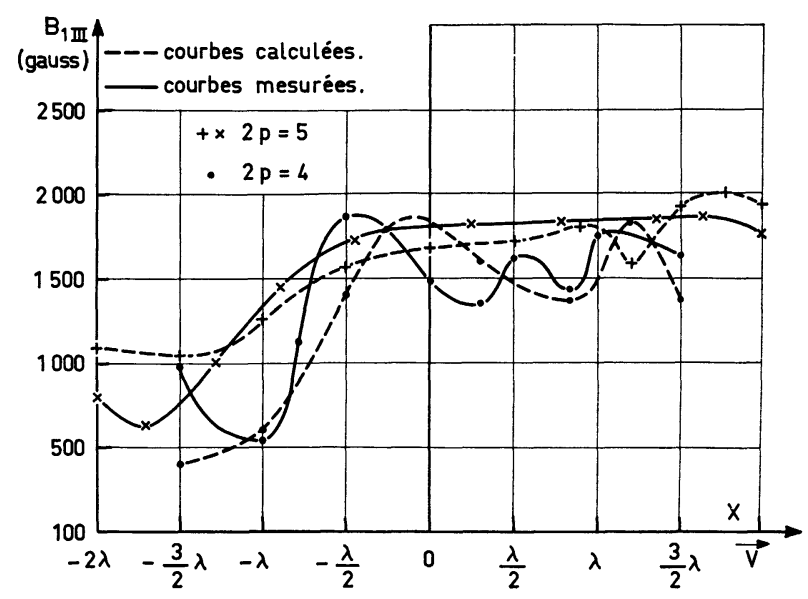

FIG. 7. 
en tenant compte des barres de fermeture, à celle relevée sur un moteur linéaire.

6. Optimalisation du rendement de la machine linéaire. - Supposons négligeables les pertes dans les enroulements d'excitation, et exprimons les puissances mises en jeu dans la machine. La puissance électrique transférée $P_{e}$ est égale au flux du vecteur de Poynting à travers la surface de séparation de l'inducteur et de l'induit.

$$
\begin{gathered}
P_{e}= \\
\left|\frac{1}{T} \int_{T} \int_{-p(\lambda / 2)}^{+p(\lambda / 2)} \int_{-c / 2}^{+c / 2}(\mathbf{E} \wedge \mathbf{H}) \mathrm{d} t \mathrm{~d} x \mathrm{~d} z\right|_{\text {pour }|y|=|a+b|}
\end{gathered}
$$

La puissance mécanique développée $P_{m}$ s'exprime en fonction du travail effectué par la force de Lorentz dans le volume de la partie active de l'induit

$$
\begin{aligned}
P_{m}=V \frac{1}{T} \int_{T} \int_{-p(\lambda / 2)}^{-p(\lambda / 2)} \int_{-a}^{+a} \int_{-c / 2}^{+c / 2} \\
\times\left(\mathbf{J}_{1} \wedge \mathbf{B}_{1}\right) \mathrm{d} t \mathrm{~d} x \mathrm{~d} y \mathrm{~d} z .
\end{aligned}
$$

Avec les approximations habituelles du calcul du transfert d'énergie dans les machines asynchrones [20], le rendement électrique d'un générateur ou d'un moteur linéaire s'écrit

$$
\eta=\left(\frac{P_{e}}{P_{m}}\right)^{ \pm 1}
$$

ou compte tenu des considérations du paragraphe 2

$$
\eta=\left|\frac{R_{e}\left\{\int_{-p(\lambda / 2)}^{+p(\lambda / 2)} \int_{-c / 2}^{+c / 2}\left[\frac{\partial}{\partial t} \int_{-p(\lambda / 2)}^{x_{1}} \bar{B}_{1} \mathrm{~d} x_{1}\right]\left(-\bar{J}_{c}^{*}\right) \mathrm{d} x \mathrm{~d} z\right\}}{V R_{e}\left\{\int_{-p(\lambda / 2)}^{+p(\lambda / 2)} \int_{-a}^{+a} \int_{-c / 2}^{+c / 2}\left(-\bar{J}_{1} \cdot \bar{B}_{1}^{*}\right) \mathrm{d} x \mathrm{~d} y \mathrm{~d} z\right\}}\right| \pm 1
$$

Cette relation se met d'après $(26)$ et $(32)$, sous la forme

$$
\eta=f\left(\bar{B}_{1} \cdot \bar{J}_{c}\right)
$$

On peut toutefois considérer $\eta$ comme une fonction de la seule variable $B_{1}$, à condition de déterminer, par synthèse de la formule (3), la distribution des courants d'excitation imposant la répartition de l'induction donnée par (26). Le principe de résolution, exposé dans [21], nous permet de calculer le courant statorique

$$
\begin{gathered}
\bar{J}_{c}=-2 \sum_{n}\left[a J_{n}\left(K_{n_{1}} \operatorname{ch} k_{n} z-1\right)+j\left(k_{n} \frac{a+b}{\mu_{1}}+\right.\right. \\
\left.\left.+\frac{1}{\mu_{3} h k_{n}}\right)\right] \cdot\left(\alpha_{n}-j \beta_{n}\right) \mathrm{e}^{j\left(\omega t-k_{n} x\right)}+ \\
+j \sum_{n} \frac{(-1)^{n p}}{\mu_{3} h k_{n}}\left(\alpha_{n}-j \beta_{n}\right) \mathrm{e}^{j \omega t}
\end{gathered}
$$

La maximalisation du rendement, qui correspond selon (41) et (42), à

$$
\frac{\partial \eta}{\partial B}=0
$$

conduit à une optimalisation, aussi bien magnétique qu'électrique, de la machine considérée. En effet, la connaissance de la distribution du champ permet de calculer, à partir de (26) et (42) les courants d'excitation et, partant, de déterminer les bobinages correspondants.

Remarquons que, si l'exploration numérique de la formule (43) ne semble présenter aucune difficulté, son analyse littérale quoique plus compliquée, est en principe possible [22].
7. Exemple d'étude des enroulements d'excitation. - L'analyse littérale de l'expression (43), effectuée dans [22], a montré que le champ purement glissant $(n=1$ dans la formule (26)) est optimal pour une machine à circuit magnétique entièrement bobiné et à nombre pair de pôles. Indiquons la manière de réaliser une telle distribution du champ dans une machine réelle de dimensions finies.

Considérons à cet effet le dispositif de la figure 5 et écrivons l'induction dans chacune des zones magnétiques sous la forme

$$
B_{1 w}=\operatorname{Re}\left\{\bar{B} \mathrm{e}^{j(\omega t-k x)}\right\} Y_{w}(x)
$$

où

$$
w=\text { I, II, III, IV et V . }
$$

Recherchons une distribution appropriée des courants d'excitation imposant aux fonctions $Y_{w}(x)$ les valeurs suivantes :

$$
Y_{\mathrm{I}}(x)=\frac{x+\left(p \frac{\lambda}{2}+m\right)}{m} Y_{\mathrm{II}}(x)=\frac{-x+\left(p \frac{\lambda}{2}+m\right)}{m}
$$

$$
Y_{\mathrm{III}}(x)=1 \quad Y_{\mathrm{IV}}(x)=Y_{\mathrm{V}}(x)=0 .
$$

D'après la relation (42), où l'on fait $n=1$, nous obtenons, après quelques calculs, la distribution des courants d'excitation dans la zone III [23].

$$
\begin{aligned}
J_{c I I I}=\left(J^{(\mathrm{I}) 2}+\right. & \left.J^{(\mathrm{II}) 2}\right)^{1 / 2} \times \\
& \times \cos \left[\omega t-k\left(x+\frac{\gamma}{k}\right)\right]+J^{(\mathrm{III})}
\end{aligned}
$$


avec

$$
\begin{aligned}
J^{(\mathrm{I})} & =-\frac{2 a B g V_{c}}{\rho_{1}}\left(K_{n_{1}} \operatorname{ch} . k z-1\right) ; \\
J^{(\mathrm{II})} & =2 B\left[\frac{(a+b) k}{\mu_{1}}+\frac{1}{\mu_{3} h k}\right] ; \\
J^{(\mathrm{III})} & =-\frac{2 B}{\mu_{3} h k} \sin \left(\omega t+k p \frac{\lambda}{2}\right) ; \\
\gamma & =\operatorname{arctg} J^{(\mathrm{II})} \cdot J^{(\mathrm{I})-1}
\end{aligned}
$$

où

$$
B=\operatorname{Re}\{\alpha-j \beta\} .
$$

La machine étant entièrement bobinée, la longueur des zones I et II tend vers zéro et, d'après (44) et (45), il faut assurer une discontinuité de l'induction à la limite des zones I-III et III-II par une distribution appropriée de courants d'excitation localisés aux points d'abscisses $X_{\mathrm{I}}$ et $X_{\mathrm{II}}$.

$$
\begin{gathered}
-p \frac{\lambda}{2}-\varepsilon<X_{\mathrm{I}}<-p \frac{\lambda}{2}+\varepsilon \\
p \frac{\lambda}{2}-\varepsilon<X_{\mathrm{II}}<p \frac{\lambda}{2}+\varepsilon .
\end{gathered}
$$

C'est ainsi que le courant $I_{\mathrm{cI}}$ en $X_{\mathrm{I}}$

$$
I_{\mathrm{cI}}=\lim _{\varepsilon \rightarrow 0} \int_{x_{\mathrm{I}}-\varepsilon}^{x_{\mathrm{I}}+\varepsilon} J_{\mathrm{cI}}(x, z, t) \mathrm{d} x
$$

est déterminé à partir de l'équation (3) appliquée au circuit magnétique de la figure 5 .

$$
\begin{aligned}
& \lim _{\varepsilon \rightarrow 0} \frac{\mu_{1}}{2(a+b)} \int_{x_{\mathrm{I}}-\varepsilon}^{x_{\mathrm{I}}+\varepsilon} J_{\mathrm{cI}}(x, z, t) \mathrm{d} x= \\
& \quad=\lim _{\varepsilon \rightarrow 0}\left\{\int_{x_{\mathrm{I}}-\varepsilon}^{x_{\mathrm{I}}+\varepsilon} \frac{\partial B_{1 \mathrm{I}}}{\partial x} \mathrm{~d} x-\frac{\mu_{1}}{\mu_{3}(a+b) h} \times\right. \\
&\left.\quad \times \int_{x_{\mathrm{I}}-\varepsilon}^{x_{\mathrm{I}}+\varepsilon} \int_{x_{\mathrm{I}}-\varepsilon}^{x_{1}} B_{1 \mathrm{I}} \mathrm{d} x \mathrm{~d} x_{1}-\frac{\mu_{1} a}{a+b} \int_{x_{\mathrm{I}}-\varepsilon}^{x_{\mathrm{I}}+\varepsilon} J_{1 z} \mathrm{~d} x\right\} .
\end{aligned}
$$

En remarquant que $B_{11}$ est égal au quotient de deux fonctions $Y_{w}(x)$ et $B \cos (\omega t-k x)$, bornées et intégrables selon Riemann et en introduisant « la fonction » de Dirac

$$
\delta(x)=\lim _{m \rightarrow 0} \frac{\partial Y_{w}(x)}{\partial x}
$$

l'expression (47) se réduit à

$\frac{\mu_{1}}{2(a+b)} I_{\mathrm{cI}}=\lim \int_{-\varepsilon-p(\lambda / 2)}^{+\varepsilon-p(\lambda / 2)} \delta(x) B \cos (\omega t-k x) \mathrm{d} x$

ou enfin

$$
I_{\mathrm{cI}}=+2 B \frac{(a+b)}{\mu_{1}} \cos \left(\omega t+k p \frac{\lambda}{2}\right) .
$$

Un raisonnement identique donne le courant $I_{\text {cII }}$ en $X_{1 I}$

$$
I_{\mathrm{cII}}=-2 B \frac{(a+b)}{\mu_{1}} \cos \left(\omega t-k p \frac{\lambda}{2}\right) .
$$

Les expressions (46) et (49) définissent le bobinage d'excitation permettant d'obtenir un champ magnétique purement glissant dans une machine linéaire à nombre pair de pôles; il doit se composer de trois parties distinctes:

- un enroulement polyphasé symétrique ordinaire dont le courant par phase vaut

$$
I_{\mathrm{cs}}=\frac{\sqrt{J^{(\mathrm{I}) 2}+J^{(\mathrm{II}) 2}}}{m \cdot n_{1}} p \lambda
$$

- un enroulement uniformément réparti, parcouru par un courant de valeur totale

$$
I_{\mathrm{cu}}=\frac{J^{(\mathrm{III})} \cdot p \lambda}{n_{\mathrm{e}}}
$$

avec $n_{\mathrm{e}}$ - nombre d'encoches de la zone III ;

- un enroulement monophasé discontinu, composé de deux bobinages situés dans des encoches d'extrémités de part et d'autre de la zone active et parcourus par les courants $I_{\mathrm{cI}}$ et $I_{\mathrm{cII}}$.

Avant d'exposer le schéma électrique d'un tel système, examinons quelques problèmes relatifs à sa conception théorique. Tout d'abord, nous avons supposé que la densité des courants d'excitation aux extrémités de la machine, qui correspond à la fonction de Dirac, est infiniment grande : en fait, on peut raisonnablement répartir les enroulements d'extrémités sur une longueur statorique finie si elle est faible devant la longueur d'onde $\lambda$. D'autre part, les courants dans l'enroulement polyphasé dépendent non seulement de $x$ et de $t$, mais aussi de $z$. Cette dépendance s'explique par l'existence de la composante $J_{1 x}$ du courant induit (32) qui entraîne une distribution bidimensionnelle du champ correctif de réaction d'induit. Ainsi pour pallier correctement la distorsion du champ résultant, il faudrait prévoir entre les conducteurs du stator des dérivations réparties sur la largeur de la carcasse magnétique. Remarquons que cette solution, technologiquement très difficile, pourrait éventuellement améliorer le rendement de la machine par intervention, dans la conversion active de l'énergie, d'une partie des connexions frontales. Le courant d'excitation dépend d'autant moins de la coordonnée $z$ que la largeur de la machine rapportée au pas polaire augmente ou encore que la résistance de ses barres de fermeture diminue; on peut déduire de (31) (32) et (46) que cette dépendance est pratiquement négligeable dans les machines où

$$
c=\lambda \text { et où } \rho_{1} \approx 4 \rho_{b} .
$$

La figure 8 représente le fragment d'un bobinage pouvant assurer un champ purement glissant dans 




FIG. 8.

la machine linéaire, la rendant ainsi magnétiquement équivalente aux machines tournantes; ce bobinage comporte :

- un enroulement triphasé alimenté par un réseau $\mathrm{A}_{1}, \mathrm{~A}_{2}, \mathrm{~A}_{3}$,

- un enroulement monophasé réparti uniformément et alimenté par l'intermédiaire d'un transformateur déphaseur $T$,

- un enroulement monophasé discontinu, alimenté par une source auxiliaire $\mathrm{A}_{0}$.

Les figures 9 et 10 font apparaître l'influence de ces enroulements, appelés « optimalisés » par opposition aux enroulements triphasés " ordinaires », sur la distribution spatiale de la valeur efficace et de la phase de l'induction $\mathbf{B}_{1}$.

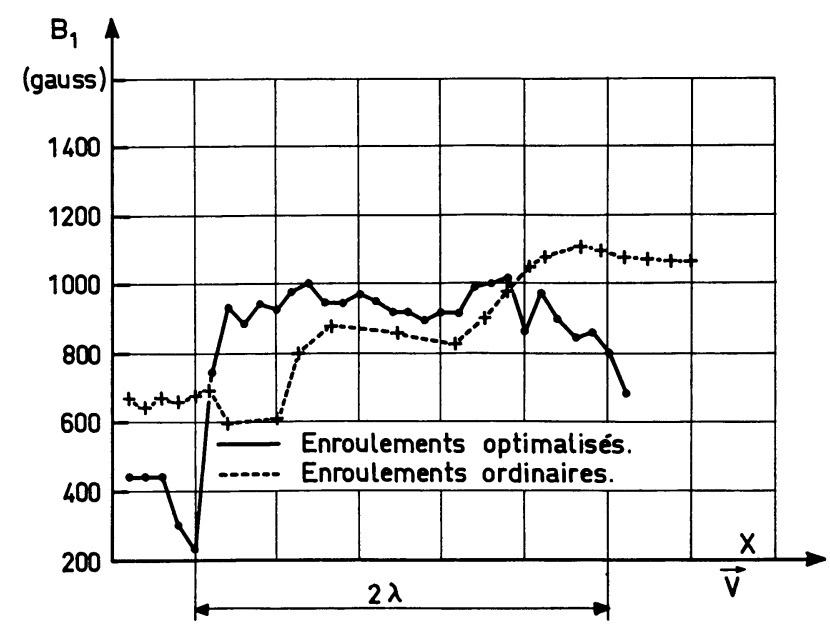

FIG. 9.

REVUE DE PHYSIQUe APPLIQUÉE. - T. 5, No 5, OCTOBRE 1970

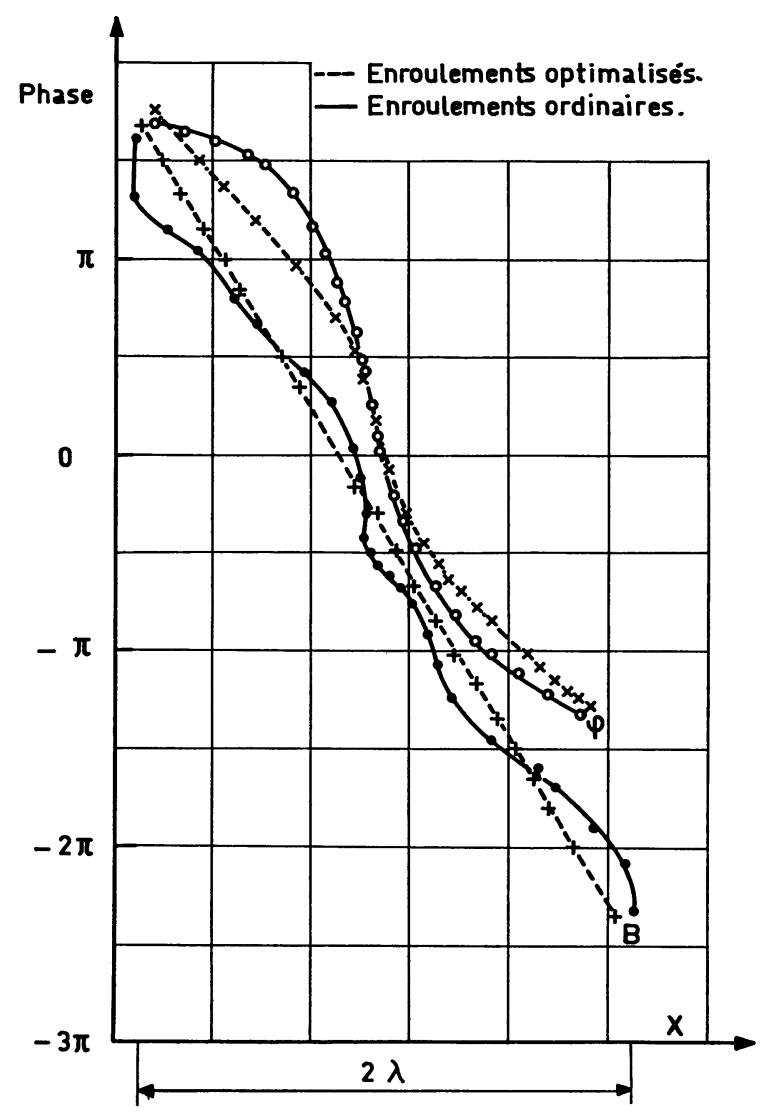

FIG. 10.

8. Modèles expérimentaux. - Afin de vérifier les études théoriques précédentes, nous avons réalisé plusieurs dispositifs linéaires dont

- le générateur magnétohydrodynamique à sodium liquide de la figure 11,

- un modèle linéaire de mesure du champ,

- le moteur linéaire de la figure 12, dont l'induit est constitué par une bande de bronze-béryllium.



FIG. 11. 


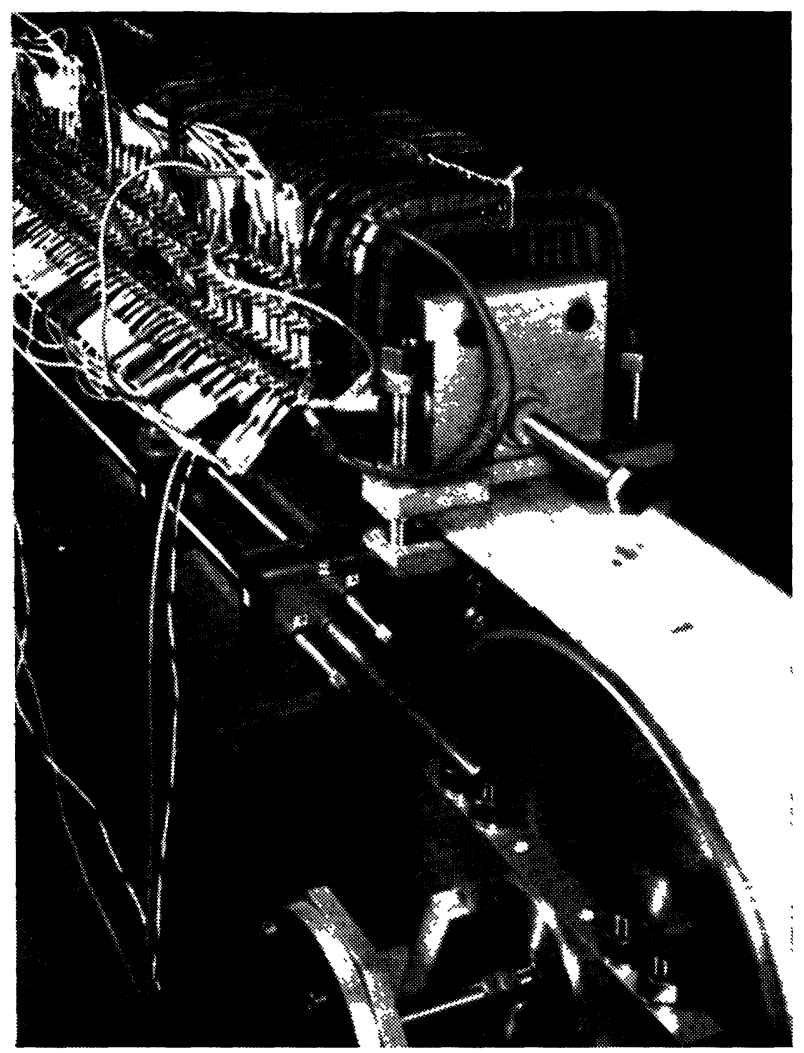

FIG. 12.

9. Conclusions. - Au terme de cet exposé, que nous avons volontairement limité à des considérations très générales afin de faire ressortir les idées directrices de nos travaux, il semble important d'insister sur la corrélation entre les recherches relatives d'une part aux moteurs linéaires et d'autre part, aux machines M. H. D. ; en effet ces deux systèmes quoique technologiquement différents sont physiquement identiques.

Nous nous proposons dans cette dernière partie de résumer très brièvement les conclusions qui se dégagent des études présentées et d'examiner les perspectives d'avenir.

L'effet de pénétration du champ est responsable de l'infléchissement des lignes de forces sur la hauteur de l'entrefer. Son action énergétique est négligeable dans la quasi-totalité des machines à induit amagnétique où la relation (16) est ordinairement satisfaite; il n'en est pas toujours de même dans les dispositifs à induit ferromagnétique, où $R_{m}$ peut atteindre des valeurs considérables.

Les effets d'extrémité entraînent une distribution du champ le long de la machine, pouvant différer considérablement de la distribution idéale, puisque se superposent à l'onde fondamentale plusieurs composantes parasites, à la fois pulsatoires et glissant à des vitesses asynchrones (20), (21), (22). Il est intéressant de remarquer que, quelle que soit la parité du nombre de pôles, la valeur efficace de l'induction se trouve affectée par les termes parasites tout le long de l'en- trefer; en particulier elle est diminuée à l'entrée et augmentée à la sortie de la machine.

L'examen de l'effet de largeur finie montre que, même dans les circuits magnétiques suffisamment longs, les perturbations dues aux effets d'extrémités se propagent à l'intérieur de la machine (35).

Il est possible, à l'aide d'un système approprié d'enroulements d'excitation (cf. 46), d'imposer, dans la zone active, un champ purement glissant, mais ceci est aisément généralisable pour une distribution quelconque du champ [18].

Aucun palliatif n'a été prévu pour supprimer l'influence des courants parasites se fermant à l'extérieur de la zone magnétique ; signalons que pour un dispositif M. H. D., on peut envisager un " feuilletage " de la veine liquide obtenu en disposant, à l'entrée et à la sortie de la machine, des lames isolantes parallèles à la direction d'écoulement du fluide [13].

Nous devons également souligner que, malgré nos efforts pour formuler théoriquement le mieux possible les phénomènes physiques, nous avons été contraints d'émettre un certain nombre d'hypothèses simplificatrices. Il est difficile de dire dans quelle mesure ces hypothèses ont idéalisé l'image du champ magnétique et, seule, une étude expérimentale très poussée peut nous éclairer à ce sujet. Signalons cependant que les calculs correspondants sont certainement plus valables pour un moteur linéaire que pour une machine $\mathrm{M}$. $\mathrm{H}$. D., dans laquelle il convient de tenir compte du profil de vitesse du fluide et de la résistivité des parois horizontales du conduit.

Pour approfondir la théorie des machines linéaires, il est indispensable de mener parallèlement des recherches théoriques et expérimentales. Il est probable en effet que l'étude théorique évoluera de la même façon que celle de la machine tournante, c'est-àdire que, partie d'un modèle physique simplifié, elle sera successivement corrigée par un certain nombre de facteurs introduits empiriquement. C'est ce point de vue qui nous a conduits à simplifier parfois les données physiques; citons, à titre d'exemple, les équations (17) et (18), où nous n'avons considéré que la forme linéaire du champ de fuites latérales, contrairement à certains auteurs qui supposent, d'ailleurs sans justification, des distributions plus complexes.

Il semble que, sur le plan théorique, les recherches doivent porter en priorité sur l'étude de la distribution du champ dans la carcasse magnétique, ce qui nécessitera une représentation plus réaliste des courants d'excitation et obligera à prendre en considération les différentes grandeurs à l'échelle du pas dentaire.

On peut remarquer enfin que l'étude de la machine linéaire à induction, loin de présenter un caractère étroitement spécialisé, permet d'envisager plusieurs " retombées " théoriques, notamment dans les domaines du couplage magnétique asynchrone et des recherches relatives aux distributions de courants dans des masses conductrices, en présence d'un champ magnétique. 


\section{Bibliographie}

[1] Shturman (G. I.), Electrichestvo no 6, 1946.

[2] Shturman (G. I.), Aronov (R. P.), Electrichestvo $\mathrm{n}^{\circ} 2,1947$.

[3] Laithwaite (E. R.), George Newnes Limited, London, 1966.

[4] VoldeCK, Nauchniye Doklady Vyshej Shkoly $\mathrm{n}^{0} 2$.

[5] VoldecK (A. I.), Izviestiya Vyshikh Uchebnikh. Zaviedieniy. Electromekanika no 1, 1959.

[6] VoldECK (A. I.), Ibid no 3, 1960.

[7] Veze (A. K.), Krumin (Ju), Magnitnaja Gidrodynamika, 1965, $\mathrm{n}^{\circ} 4$.

[8] Kant (M.), Robert (J.), Revue Générale d'Electricité, no 6, 1967.

[9] Kirko (M.), Métal liquide dans le champ magnétique. Energiya Press, Moscou, 1964.

[10] Kant (M.), C. R. Acad. Sci. Paris, Série A, 1967, 264, 773 .

[11] Kant (M.), Robert (J.), Bonnefille (R.), 8th Symposium on Engineering Aspects of Magnetohydrodynamics, Stanford, 1967.

[12] Jackson (W. D.), Pierson (E. S.), Porter (R. P.), Symposium International sur la production M. H. D. d'énergie électrique. Communication 61, Paris, 1964.

[13] Cerini (D. J.), Elliotr (D. G.), 8th Symposium on Enginereing Aspects of Magnetohydrodynamics. Stanford 1967.
[14] Kant (M.), C. R. Acad. Sci. Paris, Série A, 1968, 266, 164.

[15] Kant (M.), Bonnefille (R.), Electricity from M. H. D. International Atomic Energy Agency, Vienna 1968, III, 1717.

[16] Valdmanis (Ja. Ja.), Lilpeter (Ja. Ja.), Magnitnaja Gidrodynamika $\mathrm{n}^{0} 1,1968$.

[17] Weh (H.), Waltke (G.), Electricity from M. H. D., p. 1745, Vol. III. International Atomic Energy Agency, Vienne 1968.

[18] Kant (M.), Thèse d'Etat ès Sciences Physiques, Université de Paris 1968.

[19] Sommerfeld (A.), Electrodynamics. Academic Press, 1964.

[20] Richter (R.), Elektrische Maschinen. Tome IV, Verlag Birkhauser, 1954.

[21] Kant (M.), C. R. Acad. Sci., Paris, Série B, 1968, 266, 1455.

[22] Kant (M.), Efthymiatos (D.), C. R. Acad. Sci., Paris, 1969, 267, 1328.

[23] Bonnefille (R.), Kant (M.), 6 th Symposium of M. H. D. Institute of Physics, Academy of Science U. R. S. S., Riga 1968.

[24] Voldeck (A. I.), Lazarenko (L. F.), 6th Symposium Institute of Physics Academy of Sciences U. R. S. S., Riga 1968. 